\title{
Stroke Prevalence, Mortality and Disability-Adjusted Life Years in Children and Youth Aged 0-19 Years: Data from the Global and Regional Burden of Stroke 2013
}

\author{
Rita V. Krishnamurthi ${ }^{a}$ Gabrielle deVeber ${ }^{d}$ Valery L. Feigin ${ }^{a}$ \\ Suzanne Barker-Collo ${ }^{b}$ Heather Fullerton ${ }^{j}$ Mark T. Mackay ${ }^{f}$ Finbar O'Callahan $^{g}$ \\ M. Patrice Lindsay ${ }^{e}$ Anneli Kolk ${ }^{h}$ Warren Lol Priyanka Shah ${ }^{d}$ Alexandra Linds ${ }^{d}$ \\ Kelly Jones ${ }^{a}$ Priya Parmar ${ }^{a}$ Steve Taylor ${ }^{c}$ Bo Norrving ${ }^{i}$ George A. Mensahk \\ Andrew E. Moran ${ }^{m}$ Mohsen Naghavi ${ }^{\mathrm{n}}$ Mohammed H. Forouzanfar ${ }^{\mathrm{n}}$ \\ Grant Nguyen $^{\circ}$ Catherine O. Johnson ${ }^{\circ}$ Theo Vos ${ }^{n}$ Christopher J.L. Murray ${ }^{n}$ \\ Gregory A. Roth ${ }^{0, p}$ for the GBD 2013 Stroke Panel Experts Group

\begin{abstract}
${ }^{a}$ National Institute for Stroke and Applied Neurosciences, Auckland University of Technology, bSchool of Psychology, The University of Auckland, 'Department of Biostatistics and Epidemiology, Auckland University of Technology, Auckland, New Zealand; ${ }^{d}$ Division of Neurology, Hospital for Sick Children, ${ }^{e}$ Institute of Health Policy, Management and Evaluation, University of Toronto, Toronto, Ont., Canada; ${ }^{f}$ Department of Neurology, The Royal Children's Hospital, Melbourne, Vic., Australia; ${ }^{9}$ Institute of Child Health, University College London, London, UK; ${ }^{\mathrm{h}}$ Department of Neurology and Neurorehabilitation, Children's Clinic, Tartu University Hospital, Tartu, Estonia; 'Department of Clinical Sciences, Neurology, Lund University, Lund, Sweden; ${ }^{j}$ Benioff Children's Hospital, The University of California, San Francisco, Calif., ${ }^{k}$ Center for Translation Research and Implementation Science and Division of Cardiovascular Sciences; National Heart, Lung, and Blood Institute; National Institutes of Health, Bethesda, Md., 'Nationwide Children's Hospital,

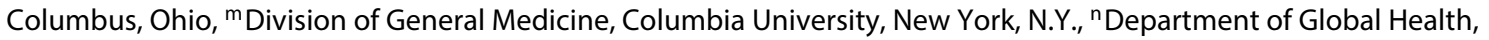
School of Medicine and Public Health, ${ }^{\circ}$ Institute for Health Metrics and Evaluation, and ${ }^{\mathrm{P} D i v i s i o n}$ of Cardiology, School of Medicine, University of Washington, Seattle, Washington, Seattle, Wash., USA
\end{abstract}

\section{Key Words}

Childhood stroke $\cdot$ Stroke epidemiology $\cdot$ Prevalence .

Deaths · Disability-adjusted life years

\begin{abstract}
Background: There is increasing recognition of stroke as an important contributor to childhood morbidity and mortality. Current estimates of global childhood stroke burden and its temporal trends are sparse. Accurate and up-to-date esti-
\end{abstract}

mates of childhood stroke burden are important for planning research and the resulting evidence-based strategies for stroke prevention and management. Objectives: To estimate the prevalence, mortality and disability-adjusted life years (DALYs) for ischemic stroke (IS), hemorrhagic stroke (HS) and all stroke types combined globally from 1990 to

Members of the GBD 2013 Stroke Panel Experts Group are listed in the Appendix at the end of the paper.

\section{KARGER 125}

(c) 2015 S. Karger AG, Basel

0251-5350/15/0453-0177\$39.50/0

E-Mail karger@karger.com

www.karger.com/ned
Rita V. Krishnamurthi

Auckland University of Technology

90 Akoranga Drive

Northcote 0627 Auckland, (New Zealand)

E-Mail rita.krishnamurthi@aut.ac.nz 
2013. Methodology: Stroke prevalence, mortality and DALYs were estimated using the Global Burden of Disease 2013 methods. All available data on stroke-related incidence, prevalence, excess mortality and deaths were collected. Statistical models and country-level covariates were employed to produce comprehensive and consistent estimates of prevalence and mortality. Stroke-specific disability weights were used to estimate years lived with disability and DALYs. Means and 95\% uncertainty intervals (Uls) were calculated for prevalence, mortality and DALYs. The median of the percent change and $95 \%$ UI were determined for the period from 1990 to 2013. Results: In 2013, there were 97,792 (95\% UI 90,564-106,016) prevalent cases of childhood IS and 67,621 (95\% UI 62,899-72,214) prevalent cases of childhood $\mathrm{HS}$, reflecting an increase of approximately $35 \%$ in the absolute numbers of prevalent childhood strokes since 1990 . There were 33,069 (95\% UI 28,627-38,998) deaths and $2,615,118$ (95\% UI 2,265,801-3,090,822) DALYs due to childhood stroke in 2013 globally, reflecting an approximately $200 \%$ decrease in the absolute numbers of death and DALYs in childhood stroke since 1990. Between 1990 and 2013, there were significant increases in the global prevalence rates of childhood IS, as well as significant decreases in the global death rate and DALYs rate of all strokes in those of age 0-19 years. While prevalence rates for childhood IS and HS decreased significantly in developed countries, a decline was seen only in $\mathrm{HS}$, with no change in prevalence rates of IS, in developing countries. The childhood stroke DALY rates in 2013 were 13.3 (95\% UI 10.6-17.1) for IS and 92.7 (95\% UI 80.5-109.7) for HS per 100,000. While the prevalence of childhood IS compared to childhood HS was similar globally, the death rate and DALY rate of HS was 6- to 7-fold higher than that of IS. In 2013, the prevalence rate of both childhood IS and HS was significantly higher in developed countries than in developing countries. Conversely, both death and DALY rates for all stroke types were significantly lower in developed countries than in developing countries in 2013. Men showed a trend toward higher childhood stroke death rates $(1.5(1.3-1.8)$ per 100,000$)$ than women $(1.1(0.9-1.5)$ per 100,000$)$ and higher childhood stroke DALY rates $(120.1$ (100.8-143.4) per 100,000) than women (90.9 (74.6-122.4) per 100,000$)$ globally in 2013. Conclusions: Globally, between 1990 and 2013, there was a significant increase in the absolute number of prevalent childhood strokes, while absolute numbers and rates of both deaths and DALYs declined significantly. The gap in childhood stroke burden between developed and developing countries is closing; however, in 2013, childhood stroke burden in terms of absolute numbers of prevalent strokes, deaths and DALYs remained much higher in developing countries. There is an urgent need to address these disparities with both global and country-level initiatives targeting prevention as well as improved access to acute and chronic stroke care.

(c) 2015 S. Karger AG, Basel

\section{Introduction}

Stroke is a significant cause of acquired brain injury and morbidity in children [1-3]. Advances in non-invasive neuroimaging have improved the detection of childhood strokes, overcoming challenges due to frequent stroke mimics in children and a lack of clinical suspicion of stroke at younger ages. The unique mechanisms underlying stroke pathophysiology in childhood remain poorly understood $[4,5]$. However, acknowledged risk factors for childhood stroke include congenital heart disease, sickle cell disease, infections, arteriopathy [6] and a number of prothrombotic conditions [7-9]. Current estimates of incidence vary from 1.3 to 13 per 100,000 , and in neonates, the incidence approximates 1 in 2,5005,000 live births $[2,10-24]$. Child mortality rates have been reported at 3.1, 0.4 and 0.2 per 100,000 for children under 1, 1-4 and 5-14 years, respectively [25]. Variations in rates across studies reflect variations in study designs and methodologies including diagnostic criteria (imaging ascertainment or not), ascertainment by International Classification of Disease (ICD) code alone or with validated diagnoses, hospital-based versus population-based settings, different age ranges captured, the inclusion of ischemic stroke (IS) and/or hemorrhagic stroke (HS), the geographical region or ethnic breakdown of the population studied and the magnitude of population studied [2]. Improved diagnosis and poststroke care may have led to an apparent increase in stroke prevalence and decline of case fatalities in children. While there have been suggestions that stroke incidence may be increasing over time, robust support for temporal changes is lacking.

Reliable epidemiological data of long-term trends, as well as the current burden of childhood stroke, are required in order to better understand the nature and impact of its burden globally and potential regional variations, as well as to inform population-level care and prevention strategies. This paper aims to describe global, region- and country-specific estimates of the prevalence, mortality, disability-adjusted life years (DALYs) and trends for IS and HS in children aged 0-19 years between 1990 and 2013 produced as part of the Global Burden of Disease (GBD) study 2013. 
Table 1. Prevalent cases, deaths and DALYs for IS and HS globally and in developing and developed countries between 1990 and 2013

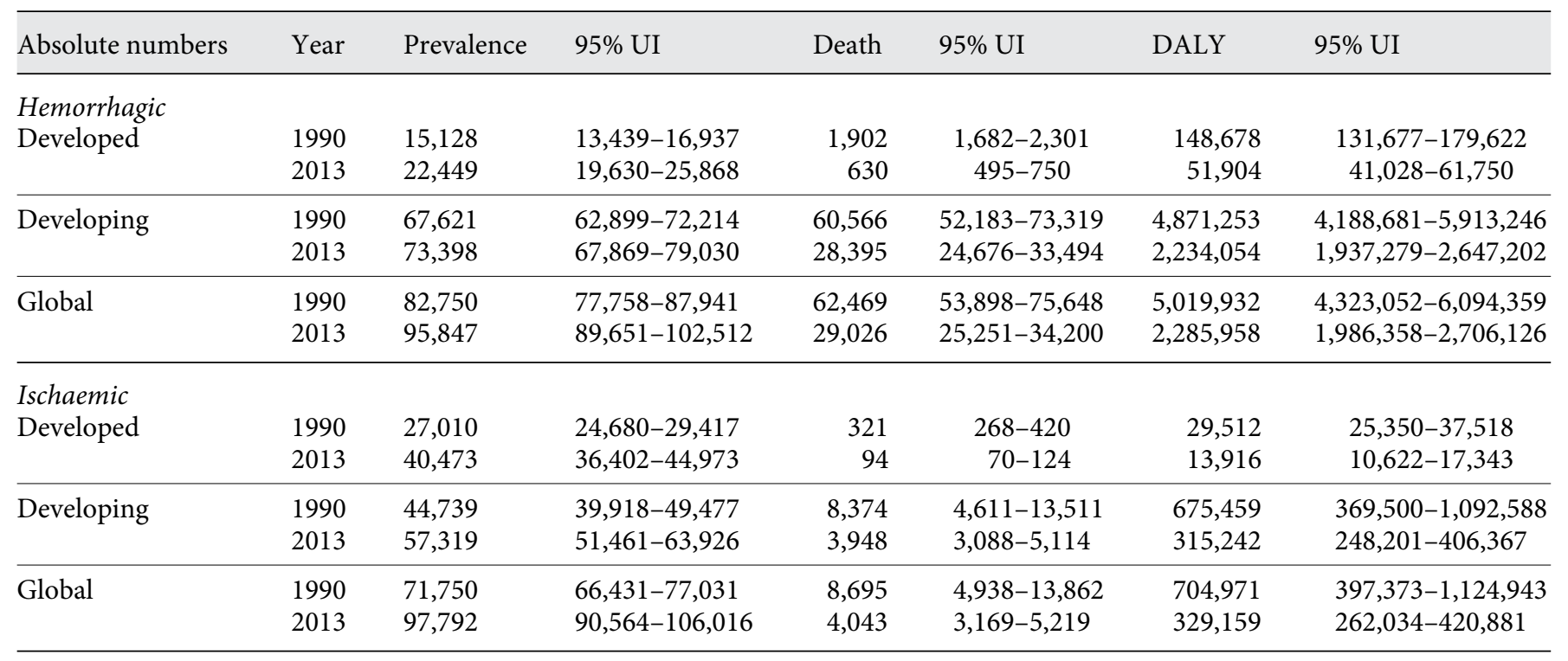

\section{Methods}

Stroke modeling and methods used to estimate all-cause mortality, cause-specific mortality, disability and disease prevalence are described elsewhere $[26,27]$. In brief, all available data on mortality were corrected for non-specific codes and used to estimate mortality rates for GBD causes of death, including IS and HS. An ensemble modeling method was used to estimate death rates by age, sex, year and country [28]. Disease prevalence was estimated using DisMod-MR disease modeling software [29] and Bayesian geospatial models. We selected estimates of stroke incidence, prevalence and case fatality from a systematic review of the scientific literature, population surveys and stroke registries as well as GBD mortality estimates. Criteria for selection were replicated from the GBD adult stroke strategy [26].

Disability due to acute stroke was considered to last for 28-30 days, while chronic stroke lasted from 30 days until death. Strokespecific disability weights were derived as part of the GBD study. DALYs were calculated as the sum of years of life lost prematurely due to stroke, based on maximum observed global longevity and years lived with disability. Countries were defined as developing or developed using GBD methods. For this analysis of childhood stroke, results were aggregated for the age group of 0-19 years, including separate estimates of death and DALY rates produced for neonates of age $0-6,7-27$ and 28-264 days.

\section{Results}

\section{Prevalence}

GBD 2013. Globally, in 2013, there were 97,792 (95\% uncertainty intervals (UIs) 89,651-102,512) prevalent cases of HS and 95,847 (95\% UI 90,564-106,016) preva- lent cases of IS among those aged $0-19$ years. Men had higher prevalence of both IS and HS than women.

Developing vs. Developed Countries. In 2013, the prevalence rate of IS was 4- to 5-fold higher in developing countries, compared to that in developed countries, while the prevalence rate for HS was approximately 2-fold higher in developing countries (table 2). Men aged 15-19 years had the highest prevalence rates for both IS and HS in both developed and developing countries at both time points (table 1).

By GBD Regions. In 2013, the lowest prevalence for IS was seen in Central Latin America, followed by Asian regions, Oceania and Andean Latin America (see online suppl. Appendix table 2; for all online suppl. material, see www.karger.com/doi/10.1159/000441087), while the highest prevalence rates for IS were seen in high-income regions of North America. The lowest prevalence of HS was seen in North Africa and Middle East, while the highest prevalence rates were seen in high-income regions of North America.

By Country. In 2013, the lowest prevalence rates were seen in Columbia, Costa Rica, El Salvador, Guatemala Honduras, Mexico, Nicaragua, Panama, Venezuela and Libya, particularly in children aged 1-4 years (online sup$\mathrm{pl}$. appendix table $1 \mathrm{a}$ and $\mathrm{b}$ ). The highest prevalence rates were seen in the United States, particularly in those aged 15-19 years.

Trends in Prevalence between 1990 and 2013. There was a significant increase in the absolute number of prev- 
Table 2. Prevalence, DALY and death rates (per 100,000) for IS and HS in 4 age groups in developing and developed countries in 1990 and 2013

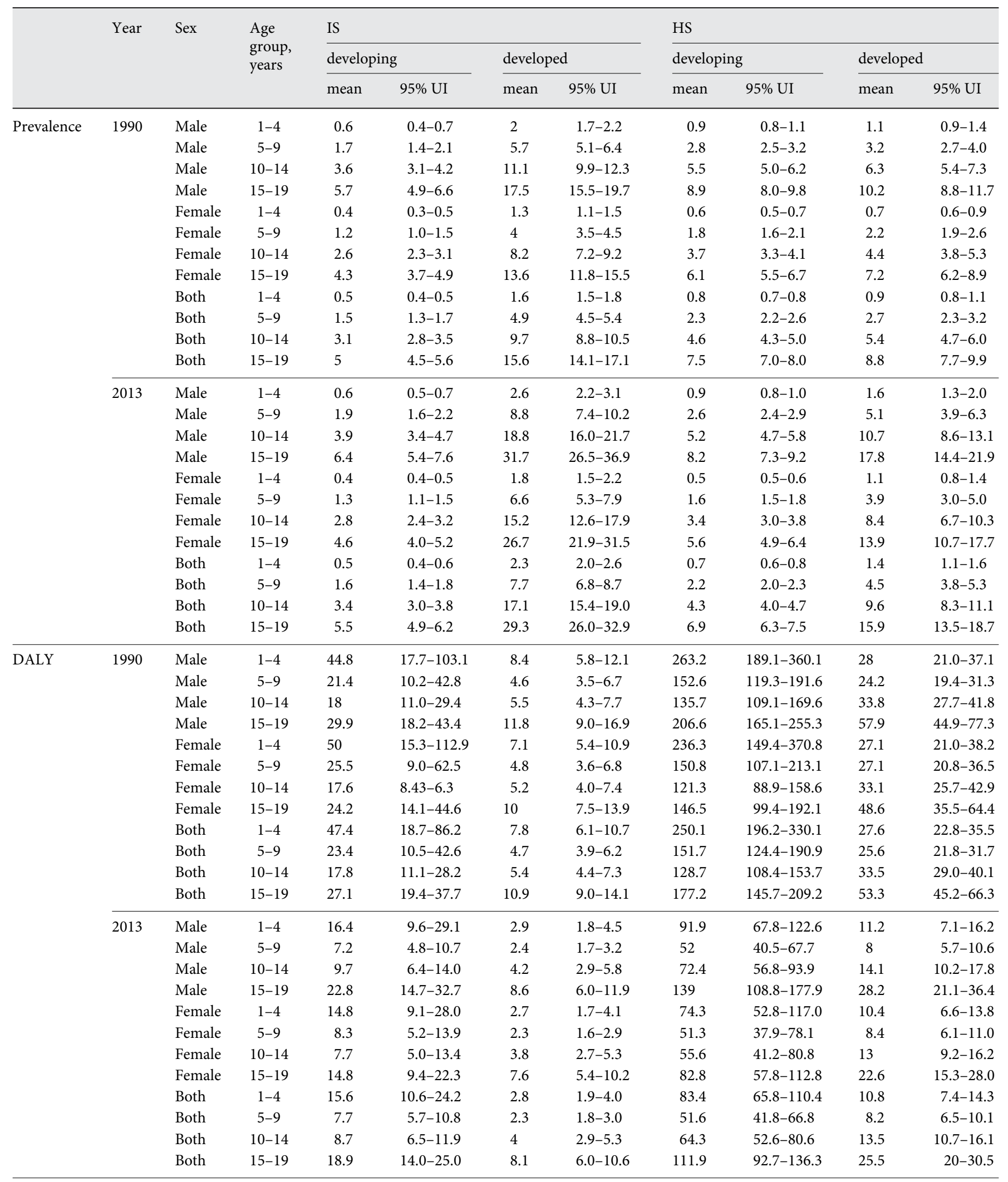


Table 2. (continued)

\begin{tabular}{|c|c|c|c|c|c|c|c|c|c|c|c|}
\hline & \multirow[t]{2}{*}{ Year } & \multirow[t]{2}{*}{ Sex } & \multirow{2}{*}{$\begin{array}{l}\text { Age } \\
\text { group, } \\
\text { years }\end{array}$} & \multicolumn{4}{|l|}{ IS } & \multicolumn{4}{|l|}{ HS } \\
\hline & & & & mean & $95 \%$ UI & mean & $95 \%$ UI & mean & $95 \%$ UI & mean & $95 \%$ UI \\
\hline \multirow[t]{20}{*}{ Deaths } & \multirow[t]{9}{*}{1990} & Male & $1-4$ & 0.5 & $0.2-1.2$ & 0.1 & $0.1-0.1$ & 3.1 & $2.2-4.3$ & 0.3 & $0.2-0.4$ \\
\hline & & Male & $5-9$ & 0.3 & $0.1-0.5$ & 0 & $0-0.1$ & 1.9 & $1.5-2.4$ & 0.3 & $0.2-0.4$ \\
\hline & & Male & $10-14$ & 0.2 & $0.1-0.4$ & 0 & $0-0.1$ & 1.8 & $1.5-2.3$ & 0.4 & $0.4-0.6$ \\
\hline & & Female & $1-4$ & 0.6 & $0.2-1.3$ & 0.1 & $0.1-0.1$ & 2.8 & $1.8-4.4$ & 0.3 & $0.2-0.5$ \\
\hline & & Female & $5-9$ & 0.3 & $0.1-0.8$ & 0.1 & $0-0.1$ & 1.9 & $1.3-2.7$ & 0.3 & $0.3-0.5$ \\
\hline & & Female & $10-14$ & 0.2 & $0.1-0.5$ & 0.1 & $0-0.1$ & 1.6 & $1.2-2.1$ & 0.4 & $0.3-0.6$ \\
\hline & & Female & $15-19$ & 0.3 & $0.2-0.6$ & 0.1 & $0.1-0.2$ & 2.1 & $1.4-2.8$ & 0.7 & $0.5-0.9$ \\
\hline & & Both & $1-4$ & 0.6 & $0.2-1.0$ & 0.1 & $0.1-0.1$ & 3 & $2.3-3.9$ & 0.3 & $0.3-0.4$ \\
\hline & & Both & $5-9$ & 0.3 & $0.1-0.5$ & 0 & $0-0.1$ & 1.9 & $1.6-2.4$ & 0.3 & $0.3-0.4$ \\
\hline & \multirow{11}{*}{2013} & Male & $5-9$ & 0.1 & $0.1-0.1$ & 0 & $0-0$ & 0.6 & $0.5-0.8$ & 0.1 & $0.1-0.1$ \\
\hline & & Male & $10-14$ & 0.1 & $0.1-0.2$ & 0 & $0-0$ & 1 & $0.8-1.3$ & 0.2 & $0.1-0.2$ \\
\hline & & Male & $15-19$ & 0.3 & $0.2-0.5$ & 0.1 & $0-0.1$ & 2 & $1.6-2.6$ & 0.4 & $0.3-0.5$ \\
\hline & & Female & $1-4$ & 0.2 & $0.1-0.3$ & 0 & $0-0$ & 0.9 & $0.6-1.4$ & 0.1 & $0.1-0.2$ \\
\hline & & Female & $5-9$ & 0.1 & $0.1-0.2$ & 0 & $0-0$ & 0.6 & $0.5-1.0$ & 0.1 & $0.1-0.1$ \\
\hline & & Female & $10-14$ & 0.1 & $0.1-0.2$ & 0 & $0-0$ & 0.7 & $0.5-1.1$ & 0.2 & $0.1-0.2$ \\
\hline & & Female & $15-19$ & 0.2 & $0.1-0.3$ & 0 & $0-0.1$ & 1.2 & $0.8-1.6$ & 0.3 & $0.2-0.4$ \\
\hline & & Both & $1-4$ & 0.2 & $0.1-0.3$ & 0 & $0-0$ & 1 & $0.8-1.3$ & 0.1 & $0.1-0.2$ \\
\hline & & Both & $5-9$ & 0.1 & $0.1-0.1$ & 0 & $0-0$ & 0.6 & $0.5-0.8$ & 0.1 & $0.1-0.1$ \\
\hline & & Both & $10-14$ & 0.1 & $0.1-0.2$ & 0 & $0-0$ & 0.9 & $0.7-1.1$ & 0.2 & $0.1-0.2$ \\
\hline & & Both & $15-19$ & 0.3 & $0.2-0.3$ & 0 & $0-0.1$ & 1.6 & $1.3-2.0$ & 0.3 & $0.2-0.4$ \\
\hline
\end{tabular}

alent cases in both developed and developing countries (table 1). Globally, and in developing countries, there was no significant change in the prevalence rates of either HS or IS between 1990 and 2013. However, in developed countries, there was a significant 2 -fold increase in prevalence rates of both IS and HS.

\section{Deaths}

GBD 2013. Globally, in 2013, there were 4,043 (95\% UI $3,169-5,219)$ deaths from IS and 29,026 (95\% UI 25,251$34,200)$ deaths from HS in the age group of $0-19$ years (table 1). Men had a trend toward a higher death rate (1.5 $(1.3-1.8)$ per 100,000$)$ than women $(1.1(0.9-1.5)$ per $100,000)$ globally in 2013 . The highest death rates among the 4 age groups were in those of 15-19 years, with the death rate of men in this age group 1.6-fold higher than it was for women. The death rate of IS was $0.2(95 \%$ UI $0.1-0.2)$ per 100,000 while the death rate from HS was 1.2 (95\% UI 1.0-1.4) per 100,000 (table 1). The age standardized death rate in children under the age of 1 year in 2013 was 6.1 (95\% UI 5.0-7.5) per 100,000, with the highest death rate seen in neonates aged $0-6$ days at 79 (95\% UI 61.6-99.2), while the DALY rate for those aged 7-27 days was 13 (95\% UI 10.4-15.7) and for those aged 28-364 days, it was 4 (95\% UI 3.2-5.3) per 100,000 (table 3).

Developing vs. Developed Countries. The absolute number of deaths across age groups in developing countries was approximately 30 - to 60 -fold higher for both IS and HS than those in developed countries. However, while 65$75 \%$ decreases in death rates was seen in developed countries across all age groups, smaller decreases of 42 and $23 \%$ were seen particularly in the 10-14 and 15-19 year age groups, respectively, in developing countries (fig. 1). In 2013, death rates in developing countries were significantly higher than in developed countries. In 1990, in developing countries, death rates for IS and HS were highest in the age group of 1-4 years; while in 2013, the highest death rates for IS and HS were seen in males of 15-19 years. In developed countries, in both 1990 and 2013, death rates for IS and HS were highest in males of age 15-19 years. 
Table 3. Global DALYs and deaths in neonates in 2013

\begin{tabular}{|c|c|c|c|c|c|c|c|c|c|}
\hline Deaths & $\begin{array}{c}0-6 \\
7-27 \\
28-364\end{array}$ & $\begin{array}{r}79.3 \\
12.8 \\
4.1\end{array}$ & $\begin{array}{c}61.6-99.2 \\
10.4-15.7 \\
3.2-5.3\end{array}$ & $\begin{array}{l}-42.4 \\
-57.6 \\
-67.5\end{array}$ & $\begin{array}{l}-53.8 \text { to }-26.9 \\
-65.0 \text { to }-45.7 \\
-73.8 \text { to }-59.3\end{array}$ & $\begin{array}{l}-41.7 \\
-57.3 \\
-67.6\end{array}$ & $\begin{array}{l}-53.4 \text { to }-25.5 \\
-64.9 \text { to }-45.1 \\
-73.8 \text { to }-59.3\end{array}$ & $\begin{array}{l}-42.4 \\
-57.5 \\
-67.5\end{array}$ & $\begin{array}{l}-53.8 \text { to }-26.8 \\
-65.0 \text { to }-45.6 \\
-73.7 \text { to }-59.2\end{array}$ \\
\hline DALYs & $\begin{array}{c}0-6 \\
7-27 \\
28-364\end{array}$ & $\begin{array}{r}6,856.3 \\
1,105.9 \\
357.8\end{array}$ & $\begin{array}{c}5,323.0-8,571.2 \\
898.0-1,359.0 \\
277.8-460.9\end{array}$ & $\begin{array}{l}-42.4 \\
-57.5 \\
-67.5\end{array}$ & $\begin{array}{l}-53.8 \text { to }-26.8 \\
-65.0 \text { to }-45.6 \\
-73.7 \text { to }-59.2\end{array}$ & $\begin{array}{l}-41.7 \\
-57.3 \\
-67.6\end{array}$ & $\begin{array}{l}-53.4 \text { to }-25.5 \\
-64.9 \text { to }-45.2 \\
-73.8 \text { to }-59.3\end{array}$ & $\begin{array}{l}-80.7 \\
-72.3 \\
-64.8\end{array}$ & $\begin{array}{l}-86.3 \text { to }-74.6 \\
-77.7 \text { to }-64.8 \\
-77.8 \text { to }-49.3\end{array}$ \\
\hline
\end{tabular}

Fig. 1. Trends in age-standardized death and DALY rates in childhood stroke between 1990 and 2013.

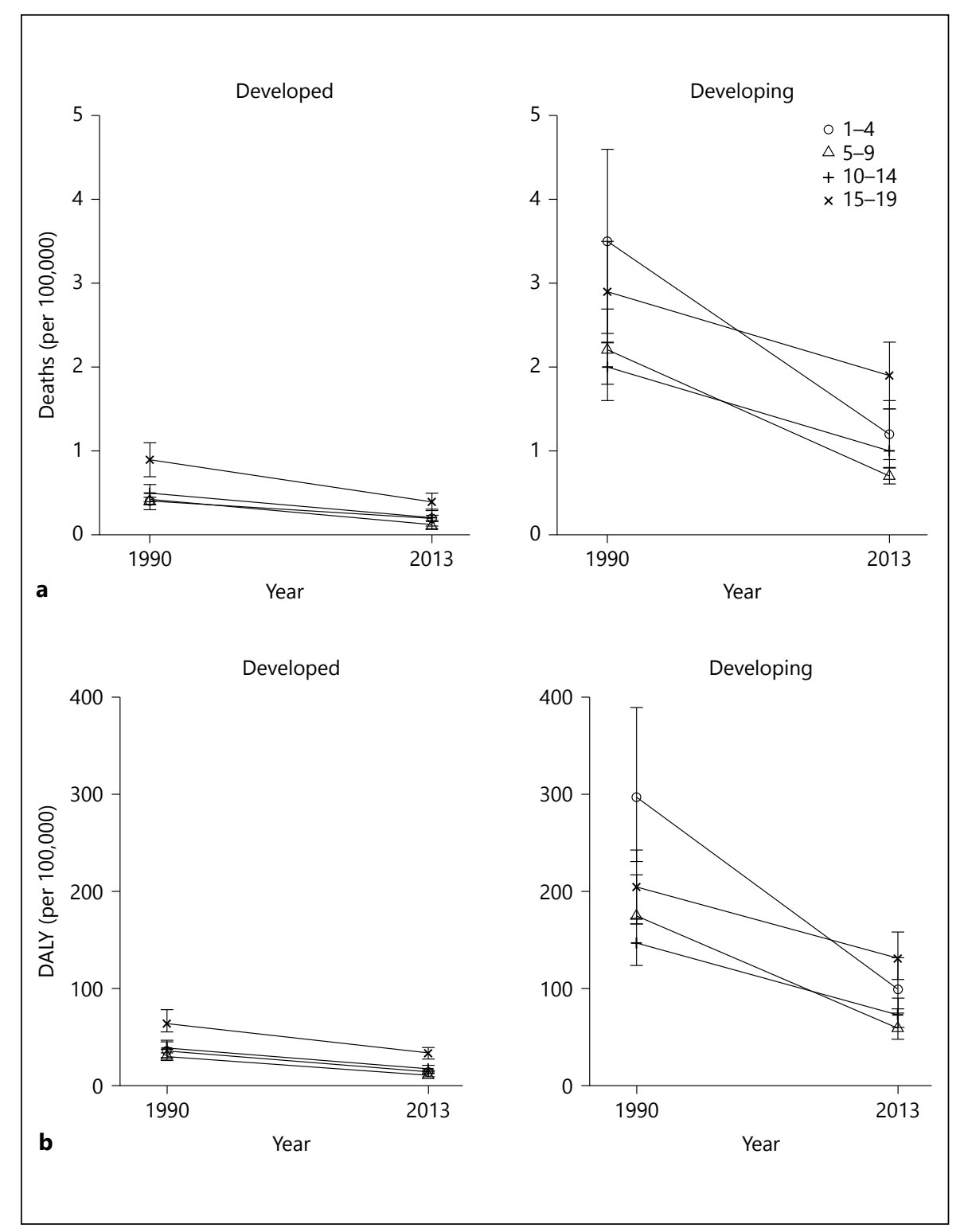


By GBD Region. According to the GBD, in 2013, death rates for IS were lowest in high-income regions of North America, Central Latin America, Central Europe and Australasia while the highest death rates were found in the Caribbean and Central Sub-Saharan Africa. The lowest death rates for HS were in Southeast Asia, Andean Latin America, Eastern Europe and Southern Latin America, while men in the high-income regions of North America, Central Asia and Tropical Latin America had the highest death rates among the GBD regions.

The lowest death rates by country for IS were seen in Taiwan, Indonesia, Malaysia, Myanmar and Vietnam across the age groups, while the highest death rates were seen in Sudan in age groups of 10-14 and 15-19 years, followed by males of 15-19 years in Sao Tome and Principe and Kiribati (online suppl. appendix table 2).

Trends in Deaths 1990-2013. There was a significant 2 -fold decline in absolute numbers of deaths in childhood stroke globally between 1990 (71,164 (95\% UI 61,12284,794)) and 2013 (33,069 (95\% UI 28,627-38,998)). Globally, there was a significant decline in death rates from all stroke types between 1990 (3.2 (95\% UI 2.7-3.8)) and 2013 (1.3 (95\% UI 1.2-2.6)) per 100,000. There was a trend toward a decline in the death rate of IS and a significant 2.3-fold decline in the death rate of HS between 1990 and 2013 (fig. 2). There was a significant decline in death rates between 1990 and 2013 in both developed and developing countries and in both men and women. The percentage change between 1990 and 2013 in the absolute numbers of HS deaths in developed countries was significantly higher than in developing countries, with a similar but non-significant trend for IS.

\section{DALYs}

In 2013, in absolute numbers, there were 2,615,118 (95\% UI 2,265,801-3,090,822) childhood stroke DALYs globally, with 329,159 (95\% UI 262,034-420,881) from IS and $2,285,958$ (95\% UI 1,986,358-2,706,126) from HS (table 1). In neonates, the DALY rates were 6,860 (95\% UI 5,325.8-8,575.7) for age 0-6 days, 1,107 (95\% UI 898.51,359.8) for age 7-27 days and 358 (95\% UI 277.9-461.2) for age 28-364 days (table 3). The DALY rate for childhood stroke was 253.5 (95\% UI 217.1-303.2) per 100,000. The DALYs rates were 13.3 (95\% UI 10.6-17.1) for IS and 92.7 (95\% UI 80.5-109.7) for HS per 100,000. Men showed a trend toward higher DALY (120.1 (95\% UI 100.8-143.4) per 100,000$)$ rates than women $(90.9$ (95\% UI 74.6-122.4) per 100,000) globally in 2013 (table 2).

Developing vs. Developed Countries. The absolute number of childhood stroke DALYs in 2013 were signifi-

Stroke Prevalence, Mortality and DALYs

in Children and Youth Aged 0-19 Years cantly higher in developing countries $(2,549,296$ (95\% UI2,206,063-3,025,321)) compared to developed countries $(65,821$ (95\% UI 53,205-77,295)). In 2013, DALY rates were also significantly higher in developing countries compared to developed countries (fig. 1; table 1) and also higher in men compared to women.

By GBD Region. The lowest DALY rates for IS were in Andean Latin America, East Asia Central Asia, Central Sub-Saharan Africa in females of 1-4 years, while the highest were in males of 10-19 years in Eastern Europe, East Asia and high-income regions of Asia Pacific. Overall, DALYs for HS were approximately 3-fold higher than those for IS. The lowest DALY rates were seen in Eastern Europe, North Africa and Middle East, Southern SubSaharan Africa and Tropical Latin America in females of 1-4 years. The highest DALY rates by GBD region were found in males in Southeast Asia, Eastern Sub-Saharan Africa, Eastern Europe and Southern Latin America.

By Country. In terms of DALY rates by country, the lowest DALY rates for IS were seen in those of 1-4 years in Iceland, followed by Lithuania and Finland (online suppl. appendix table 2). The highest DALYs were seen in Sudan in those aged 15-19 years, with high rates also seen in males of age groups of 1-4 and 5-9 years in Kiribati. Similarly, the lowest DALY rates for HS were found in Iceland in those of 1-4 years, and the highest DALYs were in Zimbabwe in the same age group.

Trends in DALYs 1990-2013. Between 1990 and 2013, the absolute number of childhood stroke DALYs declined globally ( $-54.4 \%$ (95\% UI -58.8 to -44.7$)$, but there was a greater decline in developed countries compared to developing countries. There was also a significant decline in DALY rates between 1990 and 2013 both in developed and developing countries. In developed countries, the decline in DALY rates was similar across the age groups, but in developing countries, the decline in DALYs rates was lower in adolescents (10-19 years; fig. 1). The percentage change in absolute numbers of DALYs for HS was significantly greater in developed countries, but this difference was not significant in IS (fig. 2).

\section{Discussion}

The GBD 2013 provides the most recent estimates of childhood stroke burden, as well as temporal trends in stroke burden since 1990. The overall burden of childhood stroke increased globally between 1990 and 2013 with a significant increase in the absolute number of prevalent strokes. This reflects increases in the world's popu- 


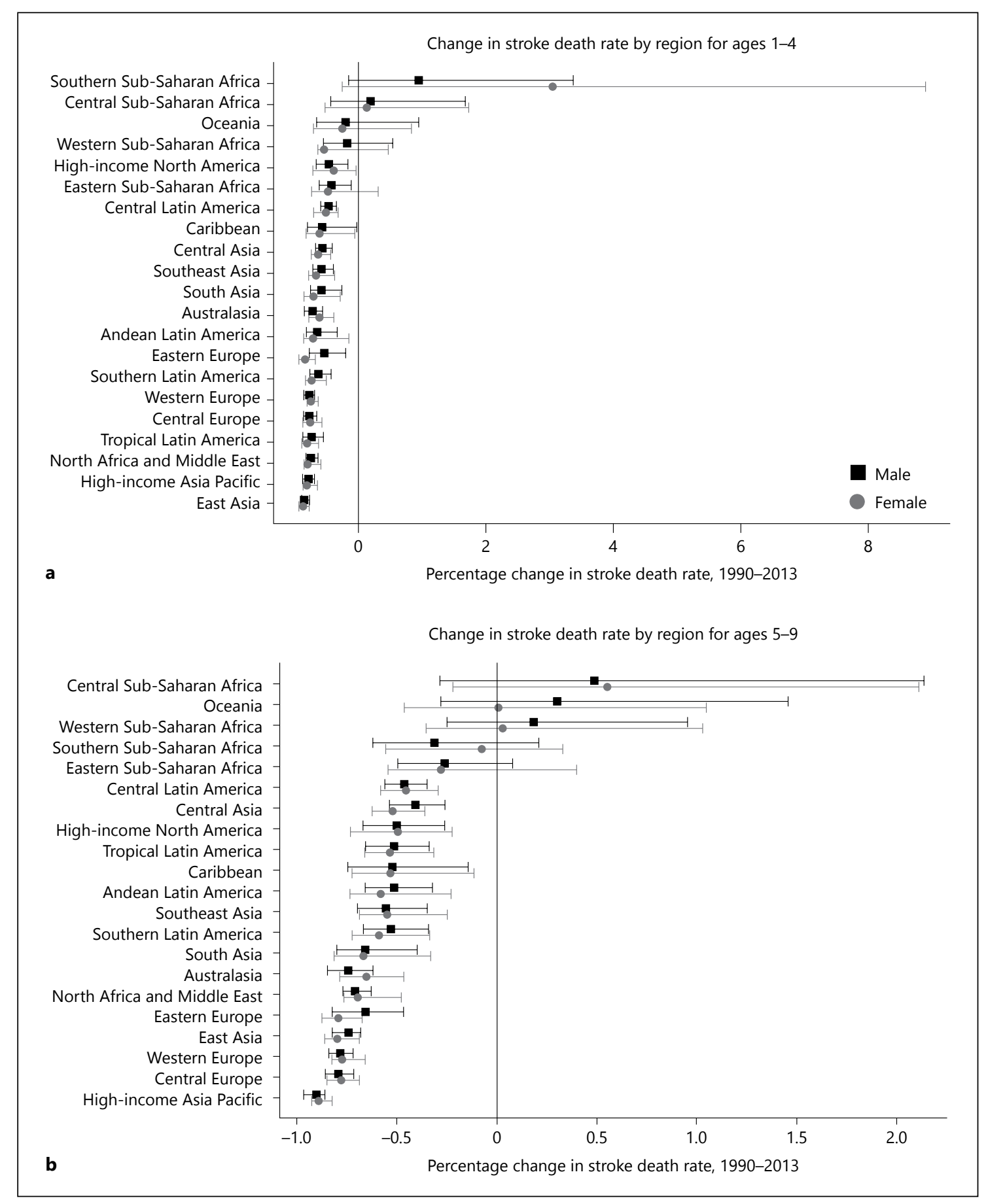

Fig. 2. Percentage change in stroke death rate, $1990-2013$ by GBD region. $\quad$ (For figures $c$ and $d$ see next page.)

lation, increased recognition and diagnosis of childhood stroke, increased survival in pediatric conditions that are complicated by stroke, and increased survival rates from childhood stroke itself. Indeed, the current estimates show that the absolute numbers of both deaths and death rates have declined significantly. However, death rates among infants below the age of 1 year remain high. While prevalence rates for IS increased between 1990 and 2013, there were significant decreases in death and DALY rates globally. This decline in childhood stroke mortality may 


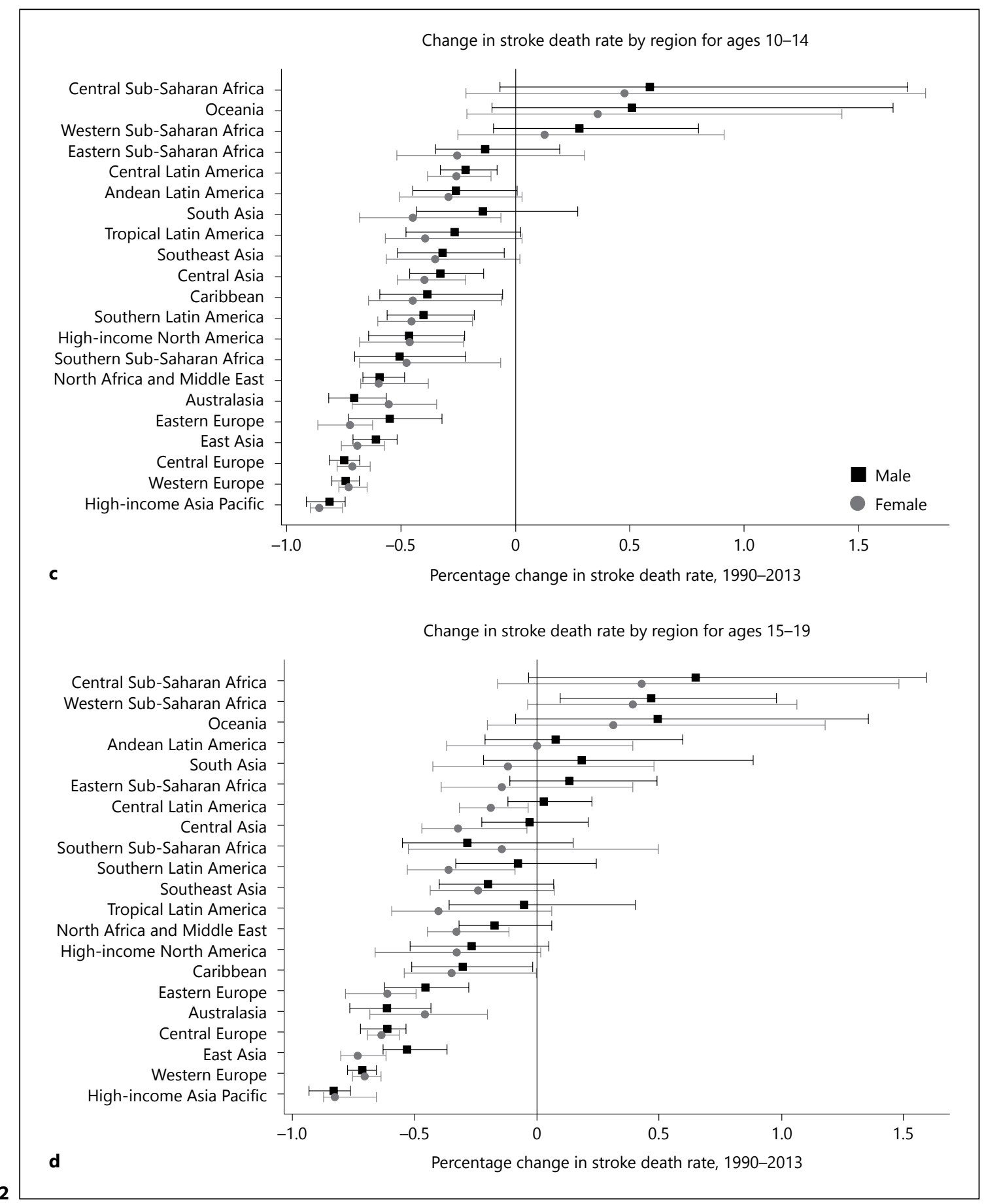

be a reflection of improving recognition [30] and poststroke medical management of childhood stroke. While recent evidence from population-based studies suggests a temporal increase in incidence rates [31,32], the differing methodologies of childhood stroke studies make it difficult to make firm conclusions or to speculate on its effects on mortality rates. However, incidence studies $[2,12,13$, 15-22, 24, 33-36] estimate IS incidence (excluding neonates) at 1.8 (SD 1.78) and HS incidence at 1.59 (SD 0.91) per 100,000. Declining rates in mortality from 1960s onward have previously been shown [10]. Interestingly, a birth cohort effect, that is, declining mortality rates with 
each successive birth cohort from 1950s onward was found, suggesting the possibility that factors around time of birth may be responsible for declining mortality rates.

In developing countries, the GBD 2013 data show that the overall burden of childhood stroke in 2013 was greater than in 1990, with higher prevalence, death rates and DALY rates of both IS and HS in such countries. The burden of stroke was highest in low-income countries and low-income GBD regions. While prevalence rates of both IS and HS in childhood stroke remained stable since 1990, these rates were higher in developing countries in comparison to developed countries in 2013. However, there were disparities in the rate of decline in deaths and DALYs in the adolescent/teen age group in developing countries. The reason for a slower decline in mortality and DALY rates in this group is unclear but could be attributed partially to racial and ethnic differences in stroke outcome. The lower decline in death rates in developing countries compared to developed countries particularly in neonates of $0-27$ days may reflect higher rates of undiagnosed stroke due to limited imaging resources, lower rates of presentation to hospital and poorer healthcare facilities in developing countries.

In 2013, the burden of HS was greater than IS; while the prevalence of IS and HS was similar, the death rate and DALY rate of HS was 6- to 7-fold higher than that of IS. The greatest number of deaths was contributed by HS, and overall burden in childhood stroke was greater in HS than IS. There is a lack of population-based estimates of stroke incidence and mortality in developing countries although some data are available [37-39], and very few that have studied temporal trends; a hospital-based study of childhood stroke showed similar proportions of HS to IS and a greater burden in men [40]. These findings reflect existing evidence on the greater burden of HS in children. Unlike in adults where IS represents approximately $80 \%$ of strokes, HS strokes can account for about $50 \%$ of the childhood strokes $[22,32]$. A study of temporal trends in long-term incidence and case fatality found that case fatality rates within 30 days, 1 and 5 years were 2 -fold higher for HS than for IS [32], while a population-based study in Denmark found that the odds of dying after HS were 1.5-fold higher than after IS [41].

The burden of stroke was greater in men than in women, particularly in those of 15-19 years, with a trend toward a higher number of absolute numbers of death and DALYs and a significantly higher death rate in men. Similar findings of higher mortality in men has also been reported elsewhere [10], although the reasons for this are unclear.
Higher death rates in developing countries are likely to be a reflection of poorer access to healthcare and lower rates of hospitalization for stroke, as well as limited availability of advanced treatment such as thrombolysis and surgical interventions. Despite this, the gap in childhood stroke burden between developed and developing countries is closing, an encouraging sign that indicates at improvements in healthcare. However, in 2013, childhood stroke burden in terms of absolute numbers of prevalent strokes, deaths and DALYs remained much higher in developing countries.

The current analysis represents the first focused pediatric stroke report from the GBD system. Age-specific estimates of both incidence and death, especially for neonates, may reflect inadequate reporting of cases. There are notable limitations to the data related to the availability and selection of published and unpublished sources. Only 2 of the 39 data sources selected for this analysis were focused studies of pediatric stroke $[31,42]$, and many pediatric studies did not meet the GBD inclusion criteria. The criteria used to select studies for GBD are the same at all ages, which poses particular challenges for estimating childhood stroke burden. For example, while many adults with stroke remain outpatients after diagnosis, most children with stroke are admitted to hospital for management. Several published manuscripts specific to childhood stroke were not included in GBD 2013 [2, 12, 13, 15-22, 24, 33-36] and may provide additional inputs for modeling as GBD data and methods focused on childhood stroke improve.

Furthermore, the diagnosis of childhood stroke based on clinical criteria alone can be particularly challenging compared with adults. Children presenting with abrupt onset focal deficits harbor non-stroke conditions in 2190\% [43] including migraine (28\%), seizures (15\%), Bell palsy (10\%), stroke (7\%) and conversion disorders (6\%) [44]. Therefore, imaging confirmation is required in pediatric stroke diagnosis unlike in adult stroke where the clinical diagnosis (not pathological diagnosis of stroke types) is based on the WHO criteria. Identification of pediatric stroke by the ICD code is more unreliable compared with adult stroke as reported in several studies. For example, Golomb et al. [45] reported that 'Code accuracy ranged from 37 to $88 \%$; each code missed more than half of arterial IS identified by the combined code search'. Agrawal et al. [12] found that ICD-9 code searches are both insensitive for pediatric stroke and carry a poor positive predictive value, and so tends to not only miss false-negative cases but also include false-positives. Much pediatric stroke research has depended on 
retrospective observational studies and administrative data sets, which have the capacity for the identification of a large number of subjects with this relatively rare disease. However, studies relying on ICD-9 codes alone for case identification are not accurate. Cases can be confirmed through chart review, although it is not usually an option for studies using administrative data sets. Radiology text string searches, although time consuming with a low yield, add significantly to the yield of screening for pediatric stroke cases [12]. In the face of these challenges specific to estimating childhood stroke, future GBD analyses may need to consider adapting the case definitions for pediatric stroke and obtaining additional unpublished study results. As childhood stroke causes vary across regions, there is also a pressing need to collect region-specific data on specific causes of childhood stroke.

In summary, globally, prevalent cases of childhood stroke have increased significantly between 1990 and 2013; however, childhood stroke death and DALY rates have declined significantly. In 2013, the overall burden of stroke was significantly higher in developing countries compared to developed countries and greater in men than in women. Childhood stroke burden was greater due to HS than IS.

Data on childhood stroke remain limited, particularly in developing countries and for neonatal stroke. Additional investment will be needed for surveillance and epidemiologic research in order to better inform policy makers facing decisions on how to invest in healthcare for children. In particular, regional disparities in childhood stroke burden will require both global and local initiatives targeted at improving access to acute and chronic stroke care for children.

\section{Acknowledgments}

This work was undertaken as a part of the GBD, Injuries and Risk Factors (GBD 2013) study. The results in this paper are prepared independently of the final estimates of the GBD 2010 study. We thank Sarah Safranek, University of Washington Health Sciences Library, for her help in developing the systematic review literature search strategies; Michael F. MacIntyre, Brittany Wurtz and Summer Ohno, University of Washington Institute for Health Metrics and Evaluation, for research coordination; Ryan M. Barber, University of Washington Institute for Health Metrics and Evaluation, for creating figures and tables for the paper; Hannah Gardener, University of Miami, for helping reviewing some studies; and Helen McDonald, AUT University National Institute for Stroke And Applied Neurosciences, for organizing teleconferences and secretarial support. The authors alone are responsible for the views expressed in this publication.

Stroke Prevalence, Mortality and DALYs in Children and Youth Aged 0-19 Years

\section{Contributions}

R.K. wrote the first draft of the report. G.R. and G.D. reviewed the first draft. V.L.F., G.A.R. and C.M. developed the study concept and oversaw the research. V.L.F., R.K. and G.A.R. undertook reviews of studies. M.H.F., M.N. and P.P. statistically analyzed the data and S.T. produced the graphs. M.H.F., M.N. and C.M. developed the statistical model and wrote a section on statistical analysis. All authors contributed to the critical revision of the manuscript for important intellectual content.

\section{Role of the Funding Source}

The study was funded by the Bill and Melinda Gates Foundation. The sponsor of the study had no role in the study design, data collection, data analysis, data interpretation or writing of the report. The writing group and GBD 2013 core analysts had access to all data sources and has responsibility for the content of the report and the decision to submit for publication.

\section{Disclaimer}

The views expressed in this article are those of the authors and do not necessarily represent the views of the National Heart, Lung, and Blood Institute, National Institute of Mental Health, National Institutes of Health, or the U.S. Department of Health and Human Services.

\section{Disclosure Statement}

All the authors declare that they have no conflict of interest.

\section{Appendix}

GBD Stroke Expert Panel Members (in Alphabetical Order by Country)

Argentina (Maria Cecilia Bahit); Australia (Amanda G. Thrift, Atte Meretoja, Bill Stavreski, Craig S. Anderson, Edwin Pearse, Geoffrey Donnan, Graeme J. Hankey, Mark T. Mackay, Stephen Davis, Zanfina Ademi); Austria (Michael Brainin); Azerbaijan (Tural Guliyev); Bahrain (Randah R. Hamadeh); Barbados (Heather Harewood, Karen Springer); Brazil (Iuri da Costa Leite, Jefferson Gomes Fernandes, Norberto Luiz Cabral, Paulo A. Lotufo); Bulgaria (Klara Dokova); Canada (Farshad Pourmalek, Gabrielle deVeber, Luciano A. Sposato, M. Patrice Lindsay, Patricia M. Riccio); Chile (Pablo M. Lavados); China (Bin $\mathrm{Li}$, Chuanhua Yu, Guohong Jiang, Jixiang Ma, Maigeng Zhou, Ming Liu, Shankuan Zhu, Wenzhi Wang, Xiaofeng Liang, Yong Zhang); Colombia (Gabriel Alcalá-Cerra), Denmark (Hanne K. Christensen, Thomas Truelsen); Egypt (Foad Abd-Allah); Ethiopia (Awoke Temesgen, Berhe Weldearegawi Sahle, Semaw Ferede Abera, Yohannes Adama Melaku), Fiji (Devina Nand); France (Maurice Giroud); Germany (Jost B. Jonas, Matthias Endres, Ronny Westerman); Greece (Konstantinos Stroumpoulis); India (Dorairaj Prabhakaran, Jeyaraj Durai Pandian, Man Mohan 
Mehndiratta, Nobhojit Roy, Panniyammakal Jeemon, Rajeev Gupta, Vasanthan Rajagopalan); Indonesia (Soewarta Kosen, Tati Suryati Warouw); Iran (Reza Malekzadeh); Ireland (Martin J. O'Donnell); Israel (Natan M. Bornstein, David Tanne); Italy (Stefano Ricci, Valeria Caso); Japan (Yoshihiro Kokubo, Yukito Shinohara); Jordan (Majed Masoud Asad); Kenya (Vitalis Kizito Bwire); Korea (Sun Ha Jee, Young-Ho Khang); Malaysia (Kim Yunjin, Ramesh Sahathevan); Mexico (Ismael Campos-Nonato); Morocco (Fortuné Gankpé); Myanmar (Chaw Yin Myint); Netherlands (Johanna M. Geleijnse); New Zealand (Priya Parmar, Rita V. Krishnamurthi, Suzanne Barker-Collo, Valery L. Feigin); Nigeria (Rufus Olusola Akinyemi); Norway (Ole Norheim); Qatar (Shams Eldin Khalifa); Russia (Michael Kravchenko, Michael Piradov, Nikolay Shalamov, Vasiliy Victorovich Vlassov, Yuri Varakin); Rwanda (Jean De Dieu Ngirabega, Jean Pierre Nyemazi, Marie Aimee Muhimpundu); Saudi Arabia (Mohammad Saeedi, Neeraj Bedi); Singapore (Narayanaswamy Venketasubramanian); South Africa (Andre Pascal Kengne); Spain (David Rojas-Rueda, Ferrán Catalá-López); Sri Lanka (Samath D.
Dharmaratne); Sweden (Bo Norrving, Rasmus Havmoeller); Uganda (Leo Atwine); United Kingdom (Amitava Banerjee, Charles Wolfe, Derrick A. Bennett, Finbar O'Callaghan, Ivy Shiue, Julia A. Critchley, Majid Ezzati, Michael Soljak, Myles D. Connor, Peter M. Rothwell, Rajiv Chowdhury, Rustam Al-Shahi Salman, William Whiteley, Zhengming Chen); Uruguay (Mercedes Colomar); USA (Adnan M. Durrani, Anand Dayama, Andrew E. Moran, Awoke Misganaw, Brett M. Kissela, Catherine Amlie-Lefond, Catherine O. Johnson, Cheng Huang, Christopher J.L. Murray, Chugh Sumeet, Daniel Kim, David K. Cundiff, David Lawrence Tirschwell, Dhruv S. Kazi, Dima Qato, Edmond Kato Kabagambe, Eric Ding, Gene Bukhman, Gene Kwan, George A. Mensah, George D. Thurston, Grant Nguyen, Gregory A. Roth, Josef Coresh, Kate Lefondulq, Kevin N. Sheth, Matthew A. Corriere, Mohammad H. Forouzanfar, Mohsen Naghavi, Nana Mainoo, Norman J. Beauchamp, Ralph L. Sacco, Richard F. Gillum, Sanjay Basu, Stephen M. Schwartz, Sumeet Chugh, Teresa Fung, Theo Vos, Tim E. Byers, Uchechukwu K.A. Sampson, Walter A. Rocca, Warren Lo).

\section{References}

1 Mallick AA, O'Callaghan FJ: Risk factors and treatment outcomes of childhood stroke. Expert Rev Neurother 2010;10:1331-1346.

2 Mallick AA, Ganesan V, Kirkham FJ, Fallon P, Hedderly T, McShane T, Parker AP, Wassmer E, Wraige E, Amin S, Edwards HB, Tilling K, O'Callaghan FJ: Childhood arterial ischaemic stroke incidence, presenting features, and risk factors: a prospective population-based study. Lancet Neurol 2014;13:35-43.

3 Mittal SO, Thatiganganna S, Kuhns B, Strbian D, Sundararajan S: Acute ischemic stroke in pediatric patients. Stroke 2015;46:e32-e34.

4 Kirton A, deVeber G: Paediatric stroke: pressing issues and promising directions. Lancet Neurol 2015;14:92-102.

5 Cárdenas JF, Rho JM, Kirton A: Pediatric stroke. Childs Nerv Syst 2011;27:1375-1390.

6 Riou EM, Amlie-Lefond C, Echenne B, Farmer M, Sébire G: Cerebrospinal fluid analysis in the diagnosis and treatment of arterial ischemic stroke. Pediatr Neurol 2008;38:1-9.

7 Armstrong-Wells J, Johnston SC, Wu YW, Sidney S, Fullerton HJ: Prevalence and predictors of perinatal hemorrhagic stroke: Results from the kaiser pediatric stroke study. Pediatrics 2009;123:823-828.

8 Bhatnagar S, Naware S, Kuber R, Thind S: Pediatric stroke: neurological sequelae in uncorrected tetralogy of fallot. Ann Med Health Sci Res 2013;3(suppl 1):S27-S30.

9 Mackay MT, Wiznitzer M, Benedict SL, Lee KJ, Deveber GA, Ganesan V; International Pediatric Stroke Study Group: Arterial ischemic stroke risk factors: the international pediatric stroke study. Ann Neurol 2011;69:130-140.

10 Mallick AA, Ganesan V, O'Callaghan FJ Mortality from childhood stroke in England and Wales, 1921-2000. Arch Dis Child 2010; 95:12-19.
11 Benders MJ, Groenendaal F, Uiterwaal CS, Nikkels PG, Bruinse HW, Nievelstein RA, de Vries LS: Maternal and infant characteristics associated with perinatal arterial stroke in the preterm infant. Stroke 2007;38:1759-1765.

12 Agrawal N, Johnston SC, Wu YW, Sidney S, Fullerton HJ: Imaging data reveal a higher pediatric stroke incidence than prior US estimates. Stroke 2009;40:3415-3421.

13 Hall R, Khan F, O'Callaghan C, et al: Ontario Stroke Evaluation Report: Prescribing System Solutions to Improve Stroke Outcomes. Toronto, Institute for Clinical Evaluative Sciences, 2012.

14 Salih MA, Abdel-Gader AG, Al-Jarallah AA, Kentab AY, Alorainy IA, Hassan HH, Bahakim HM, Kurbaan KM, Zahraa JN, Al-Nasser MN, Nasir AA, Khoja WA, Kabiraj MM: Stroke in Saudi children. Epidemiology, clinical features and risk factors. Saudi Med J 2006; 27(suppl 1):S12-S20.

15 Al-Sulaiman A, Bademosi O, Ismail H, Magboll G: Stroke in Saudi children. J Child Neurol 1999;14:295-298.

16 Fullerton HJ, Wu YW, Sidney S, Johnston SC: Recurrent hemorrhagic stroke in children: a population-based cohort study. Stroke 2007; 38:2658-2662.

17 Simma B, Martin G, Müller T, Huemer M: Risk factors for pediatric stroke: consequences for therapy and quality of life. Pediatr Neurol 2007;37:121-126.

18 Steinlin M, Pfister I, Pavlovic J, Everts R, Boltshauser E, Mori AC, Mercati DG, Hänggeli CA, Keller E, Luetschg J, Marcoz J, Ramelli GP, Perez ER, Schmitt-Mechelke T, Weissert $\mathrm{M}$ : The first three years of the swiss neuropaediatric stroke registry (SNPSR): a populationbased study of incidence, symptoms and risk factors. Neuropediatrics 2005;36:90-97.
19 Williams A, Kirkham F: Childhood stroke, stroke-like illness and cerebrovascular disease. Stroke 2004;35:311.

20 Barnes C, Newall F, Furmedge J, Mackay M, Monagle P: Arterial ischaemic stroke in children. J Paediatr Child Health 2004;40:384387.

21 Chung B, Wong V: Pediatric stroke among Hong Kong Chinese subjects. Pediatrics 2004; 114:e206-e212.

22 Fullerton HJ, Wu YW, Zhao S, Johnston SC: Risk of stroke in children: ethnic and gender disparities. Neurology 2003;61:189-194.

23 Lynch JK, Hirtz DG, DeVeber G, Nelson KB: Report of the national institute of neurological disorders and stroke workshop on perinatal and childhood stroke. Pediatrics 2002;109: 116-123.

24 Merino Arribas JM, de Pablo Chinchetru R, Grande Grande T, Sánchez Martín J, González de la Rosa JB: [Nontraumatic hemorrhagic stroke in children after the neonatal period]. An ESP Pediatr 1997;47:392-396.

25 Miniño AM, Murphy SL, Xu J, Kochanek KD: Deaths: final data for 2008. Natl Vital Stat Rep 2011;59:1-126.

26 Roth GA, Johnson CO, Nguyen G, Naghavi $\mathrm{M}$, Feigin VL, Murray CJL, Forouzanfar $\mathrm{MH}$, Vos T: Methods for estimating the global burden of cerebrovascular diseases. Neuroepidemiology 2015;45:146-151.

27 Feigin VL, Krishnamurthi R, Parmar P, Norrving $B$, Mensah GA, Bennett DA, BarkerCollo S, Moran AE, Sacco RL, Truelsen T, Davis S, Pandian JD, Naghavi M, Forouzanfar MH, Nguyen G, Johnson C, Vos T, Meretoja A, Murray CJL, Roth GA: Update on the global burden of ischemic and hemorrhagic stroke in 1990-2013: the GBD 2013 study. Neuroepidemiology 2015;45:161-176. 
28 GBD 2013 Mortality and Causes of Death Collaborators: Global, regional, and national age-sex specific all-cause and cause-specific mortality for 240 causes of death, 1990-2013: a systematic analysis for the global burden of disease study 2013. Lancet 2015;385:117-171.

29 Flaxman AD, Vos T, Murray CJL: An Integrative Metaregression Framework for Descriptive Epidemiology, 2015.

30 DeVeber G: Stroke and the child's brain: an overview of epidemiology, syndromes and risk factors. Curr Opin Neurol 2002;15:133-138.

31 Mallick AA, O'Callaghan FJ: The epidemiology of childhood stroke. Eur J Paediatr Neurol 2010;14:197-205.

32 Gandhi SK, McKinney JS, Sedjro JE, Cosgrove NM, Cabrera J, Kostis JB: Temporal trends in incidence and long-term case fatality of stroke among children from 1994 to 2007. Neurology 2012;78:1923-1929.

33 Zahuranec DB, Brown DL, Lisabeth LD, Morgenstern LB: Is it time for a large, collaborative study of pediatric stroke? Stroke 2005;36 1825-1829.

34 Lynch JK, Hirtz DG, DeVeber G, Nelson KB: Report of the national institute of neurological disorders and stroke workshop on perinatal and childhood stroke. Pediatrics 2002;109: 116-123.
35 Williams AN, Eunson PD, McShane MA, Lynn R, Green S, Kirkham F: Childhood cerebrovascular disease and stroke like illness in the United Kingdom and eire. A descriptive epidemiological study. Arch Dis Child 2002; 86(suppl 1):A40-A41.

36 deVeber G; Canadian Paediatric Ischemic Stroke Study Group (2000a): Canadian pediatric ischemic stroke registry: analysis of children with arterial ischemic stroke (abstract). Ann Neurol 2000;48:526.

37 Lavados PM, Sacks C, Prina L, Escobar A, Tossi C, Araya F, Feuerhake W, Galvez M, Salinas R, Alvarez G: Incidence, 30-day case-fatality rate, and prognosis of stroke in Iquique, Chile: a 2-year community-based prospective study (PISCIS project). Lancet 2005;365: 2206-2215.

38 Azarpazhooh MR, Etemadi MM, Donnan GA, Mokhber N, Majdi MR, Ghayour-Mobarhan M, Ghandehary K, Farzadfard MT, Kiani R, Panahandeh M, Thrift AG: Excessive incidence of stroke in Iran: evidence from the mashhad stroke incidence study (MSIS), a population-based study of stroke in the middle east. Stroke 2010;41:e3-e10.
39 Kalita J, Goyal G, Misra UK: Experience of pediatric stroke from a tertiary medical center in North India. J Neurol Sci 2013;325:67-73.

40 Deng Y, Wang Y, Yang W, Yu Y, Xu J, Wang $\mathrm{Y}, \mathrm{Gao} \mathrm{B}$ : Risk factors and imaging characteristics of childhood stroke in China. J Child Neurol 2015;30:339-343.

41 Statler KD, Dong L, Nielsen DM, Bratton SL: Pediatric stroke: clinical characteristics, acute care utilization patterns, and mortality. Childs Nerv Syst 2011;27:565-573.

42 Giroud M, Lemesle M, Gouyon JB, Nivelon JL, Milan C, Dumas R: Cerebrovascular disease in children under 16 years of age in the city of Dijon, France: a study of incidence and clinical features from 1985 to 1993 . J Clin Epidemiol 1995;48:1343-1348.

43 Shellhaas RA, Smith SE, O'Tool E, Licht DJ, Ichord RN: Mimics of childhood stroke: characteristics of a prospective cohort. Pediatrics 2006;118:704-709.

44 Mackay MT, Chua ZK, Lee M, Yock-Corrales A, Churilov L, Monagle P, Donnan GA, Babl FE: Stroke and nonstroke brain attacks in children. Neurology 2014;82:1434-1440.

45 Golomb MR, Garg BP, Saha C, Williams LS: Accuracy and yield of ICD-9 codes for identifying children with ischemic stroke. Neurology 2006;67:2053-2055. 